\title{
Composition-dependent stability of the medium-range order responsible for metallic glass formation
}

\author{
Feng Zhang, ${ }^{1,2}$ Min Ji, ${ }^{1,2}$ Xiao-Wei Fang, ${ }^{1,2,3}$ Yang Sun,${ }^{1,2}$ Cai-Zhuang Wang, ${ }^{1,2}$ \\ Mikhail I. Mendelev, ${ }^{1}$ M. J. Kramer, ${ }^{1,4}$ Ralph E. Napolitano, ${ }^{1,4}$ Kai-Ming Ho ${ }^{1,2}$ \\ ${ }^{1}$ Ames Laboratory, US Department of Energy, Ames, Iowa 50011, USA \\ ${ }^{2}$ Department of Physics and Astronomy, Iowa State University, Ames, Iowa 50011,
} USA

${ }^{3}$ Hefei National Laboratory for Physical Sciences at Microscale and Department of Physics, University of Science and Technology of China, Hefei, Anhui 230026, People's Republic of China

${ }^{4}$ Department of Materials Science and Engineering, Iowa State University, Ames, Iowa 50011, USA

\begin{abstract}
The competition of characteristic medium range order corresponding to amorphous alloys and those in ordered crystalline phases is central to phase selection and morphology evolution under various processing conditions. We examine the stability of a model glass system, $\mathrm{Cu}-\mathrm{Zr}$, by comparing the energetics of various medium-range structural motifs over a wide range of compositions using first-principles calculations. We focus specifically on motifs that represent possible building blocks for competing glassy and crystalline phases, and we employ a genetic algorithm to efficiently identify the energetically favored decorations of each motif for specific compositions. Our results


show that a Bergman-type motif with crystallization-resisting icosahedral symmetry is energetically most favorable in the composition range $0.63<x_{\mathrm{Cu}}<0.68$, and is the underlying motif for one of the three optimal glass-forming ranges observed experimentally for this binary system [1]. This work establishes an energy-based methodology to evaluate specific medium-range structural motifs which compete with stable crystalline nuclei in deeply undercooled liquids.

Key words: metallic glass; medium-range order; genetic algorithm. 


\section{Introduction}

Metallic glasses are typically formed in bulk by rapid cooling from liquid melts. Within specific compositions [2], unstable or metastable structures may be effectively stabilized by extremely sluggish relaxation kinetics. A large number of such metallic glasses have been identified, and found applications because of their superior mechanical stiffness, strength, and chemical stability relative to their crystalline counterparts. Various phenomenological rules have been formulated to interpret the glass formability for metallic systems [3-6]. However, atomistic modeling of amorphous alloys is challenging due to the lack of periodic order. While thermodynamic stability of crystalline intermetallic phases can be readily and accurately described because of their well-defined crystalline order, for disordered systems, simulations on much larger time and length scales are required for proper statistical averaging.

The composition-dependent stability of metallic glass is poorly understood within existing theoretical framework. Some thermodynamic models predict the glass-forming composition range by comparing the energy of the disordered glassy state against the energy of a solid solution in the solvent lattice [7, 8]. However, a more relevant comparison can be made using complex intermetallic compounds as the reference state [9], especially when the solute and solvent have large size disparities or the solute concentration is high. Moreover, most of this work is empirical and not based on accurate energetic calculations, which is required to reliably establish phase stability. In addition, existing first-principles modeling of metallic glass is mainly based on ab-initio molecular dynamics (AIMD), whose capabilities are limited because of a large mismatch between experimental and computational time scales: Experimental melt-quenching time is usually

of the order of milliseconds: orders of magnitude longer than what can be achieved in 
AIMD. This mismatch results in significantly under-evolved structural configurations in computer simulations.

An emerging consensus is that metallic glass displays short to medium range order arising from the packing of local clusters [10-19]. This suggests that the stability of certain local motifs can be used to characterize the energetic stability of the glassy system. In this way, the energetic stability of a metallic glass can be estimated without a priori detailed knowledge of the amorphicity of the glass. Rather than focusing on a single detailed solution to the structure, which is not unique, our approach aims to capture the essential components of local order, which collectively govern the overall stability and physical properties of the bulk glass. Such a treatment also results in a significant reduction in the size of the calculated system, allowing the use of accurate first-principles methods.

\section{Model and Simulation Details}

\subsection{Medium-range structural motifs in the $\mathrm{Cu}-\mathrm{Zr}$ system}

As an example, we examine the relative stability of various structural motifs in the $\mathrm{Cu}-\mathrm{Zr}$ system which displays strong glass formability for a wide range of compositions [1, 20-23]. The $\mathrm{Cu}-\mathrm{Zr}$ system has three optimal glass-forming regions with the $\mathrm{Cu}$ composition $\left(x_{\mathrm{Cu}}\right)$ around $0.50,0.56$ and 0.64 [1]. The composition-dependent glass formability has been attributed to the density of the amorphous phase [1], or a rationally designed parameter integrating the mixing enthalpy, the driving force for decomposition, and the excessive atomic space [24]. However, neither of these approaches fully accounts for the chemical and topological medium-range order responsible for glass formation. 
We consider both crystallization-favoring and crystallization-resisting motifs. Motifs collected from known $\mathrm{Cu}-\mathrm{Zr}$ compounds are labeled as crystallization-favoring motifs. For the $\mathrm{Cu}-\mathrm{Zr}$ system, simulations have demonstrated that local icosahedral clustering correlates with glass formation from the liquid state for certain compositions [10, 15, 17]. Because of the five-fold symmetry, the icosahedral cluster is not a space-filling motif, and is geometrically frustrating for crystalline packing. Therefore, icosahedral motifs are regarded as crystallization-resisting motifs. The effects of pentagonal short-range order with the five-fold symmetry on glass transition were examined in a two-dimensional toy model, using an interatomic potential artificially designed to favor the pentagonal packing [25]. Here, we focus on a real material, making no pre-assumption on the nature of the interactions in the system. In addition, we consider not only the first atomic shell (short-range order), but the next several atomic shells in order to include the full medium-range order [14]. For energy stability studies, these motifs need to be embedded in an atomic network, since the stability of stand-alone clusters is dominated by unsaturated atoms at the surface, which are irrelevant in well-packed solids. The atomic network is chosen to be periodic to facilitate computation. For crystalline-favoring motifs, all known stable and metastable structures in the phase diagram are tested. For icosahedral motifs, we choose quasicrystal approximant structures representing several typical medium-range icosahedral order [26, 27]. The physical and chemical properties of the approximant structures highly resemble those of the quasicrystals with the same local icosahedral clusters, suggesting that these local clusters play a dominant role in determining the properties of such aperiodic materials. Therefore, the approximant structures should be good descriptors for metallic glasses with strong local icosahedral order as well. 
The crystalline-favoring motifs considered are extracted from the compounds $\mathrm{CuZr}_{2}$ (I4/mmm), $\mathrm{CuZr}(\mathrm{Pm} 3 \mathrm{~m}), \mathrm{Cu}_{10} \mathrm{Zr}_{7}$ (Aba2), $\mathrm{Cu}_{8} \mathrm{Zr}_{3}$ (Pnma), $\mathrm{Cu}_{51} \mathrm{Zr}_{14}(\mathrm{P} 6 / \mathrm{m})$, and $\mathrm{Cu}_{5} \mathrm{Zr}$ $(\mathrm{F} \overline{4} 3 \mathrm{~m})$ [28]. The space group nomenclature is given in the brackets, which establishes the packing feature of each motif based on the details of the atomic decoration for each compound. In addition, four icosahedral motifs are included, which are derived from quasicrystal approximants $\operatorname{Mg}_{32}(\mathrm{Al}, \mathrm{Zn})_{49}[29], \quad \alpha(\mathrm{AlMnSi})[30], \quad \mathrm{YbCd}_{6}[31]$ and $\mathrm{MoAl}_{12}$ [32], as shown in Fig. 1 (a), (b), (c) and (d), respectively. $\mathrm{Mg}_{32}(\mathrm{Al}, \mathrm{Zn})_{49}, \mathrm{YbCd}_{6}$ and $\mathrm{MoAl}_{12}$ all have bcc lattices, and $\alpha(\mathrm{AlMnSi})$ also has a nearly bcc structure. Thus, the structures in Fig. 1 are bounded by the Wigner-Seitz unit cell of the bcc lattice, which has the shape of a truncated octahedron. Figure 1 (a), (b), (c) represent the Bergman-type [29], Mackay-type [33], and Tsai-type [34] icosahedral motifs, respectively. The icosahedral motif shown in Fig. 1 (d) will be referred to as MoAl-type, whose characteristic feature is a network of non-interpenetrating icosahedra.

\subsection{Iterative genetic-algorithm (GA) search}

We study the relative stability of these crystalline-favoring motifs and icosahedral motifs for a wide range of $\mathrm{Cu}$ compositions $\left(0.25<x_{\mathrm{Cu}}<0.85\right)$. For each motif, the Bravais lattice and the atomic sites are predetermined by a template structure as discussed above. What remains to be decided is the energetically most favorable decoration of the template with $\mathrm{Cu}$ and $\mathrm{Zr}$ atoms according to a specific $x_{\mathrm{Cu}}$. We use a global GA search $[35,36]$ to perform this task, which is carried out separately for each motif at each composition. The initial pool for the GA search contains a number of structures that share the same template and composition but have randomly generated arrangements of $\mathrm{Cu}$ and 
$\mathrm{Zr}$ atoms. The energy of each structure is evaluated as its fitness. The structures are allowed to relax during the energy calculations. While these relaxations create some geometric distortions, the key topological features of the motif will be maintained. Then, "parents" are selected primarily from structures with relatively low energies to create next-generation structures based on a "cut-and-paste" mating operation, as schematically illustrated in Fig. 2 [35, 36]. We use the unrelaxed structures representing different decorations during the mating process. The structures with the highest energies in the pool will be replaced by the next-generation structures that have better fitness values. The above process is repeated until the structure pool stops evolving.

For each motif, the GA search is carried out in unit cells with about 100 atoms, allowing us to vary the composition of the system with a fine interval of approximately one percent. Such large unit cells are time-consuming if first-principles calculations are used throughout the search. Thus it is necessary to adopt a more efficient auxiliary potential for energy calculations during the GA search. Here, we choose an interatomic potential in the Finnis-Sinclair (FS) form [37], that has been commonly used to model the $\mathrm{Cu}-\mathrm{Zr}$ system [38]. The FS potential can be expressed as

$$
U=\sum_{i<j} \phi^{\alpha_{i} \alpha_{j}}\left(r_{i j}\right)+\sum_{i} \Phi^{\alpha_{i}}\left(\rho_{i}\right)
$$

In Eq. $1, \alpha^{i}$ denotes the type of atom $i$, and $r_{i j}$ is the separation between atoms $i$ and $j . \phi^{\alpha_{i} \alpha_{j}}\left(r_{i j}\right)$ is the energy for the pairwise interaction between atoms $i$ and $j$, and $\Phi^{\alpha_{i}}\left(\rho_{i}\right)$ is the energy for embedding an atom with type $\alpha_{i}$ at a position with a background charge density $\rho_{i}$, which can be further expressed as 


$$
\rho_{i}=\sum_{j} \psi^{\alpha_{i} \alpha_{j}}\left(r_{i j}\right)
$$

where $\psi^{\alpha_{i} \alpha_{j}}$ represents the contribution of atom $j$ to the background charge density at the position of atom $i$.

While the FS potential is sufficiently fast, it was developed to fit a broad range of thermodynamic parameters [38]. We check the FS potential with more accurate density functional theory (DFT), as implemented in the VASP package $[39,40]$. The generalized gradient approximation proposed by Perdew, Burke, and Ernzerhof [41] is used for the exchange-correlation functional. The electron-ion interaction is treated with the projector-augmented wave method [42]. A cut-off energy of $400 \mathrm{eV}$ is used for the plane-wave basis. The total-energy converges to $10^{-5} \mathrm{eV}$ per cell in each self-consistent loop, and the structural relaxation terminates when the force on each atom is smaller than $0.01 \mathrm{eV} / \AA$.

Fig. 3 (a) compares the formation energies ( $E_{\text {form }}$ ) calculated by FS and DFT for structures collected at $x_{\mathrm{Cu}}=0.65$ for all structural motifs. $E_{\text {form }}$ is defined as the total energy of a structure referenced to the phase separated mixture of pure $\mathrm{Cu}$ and pure $\mathrm{Zr}$. As one can see, there exist significant disagreements between the relative stability of the motifs calculated by DFT and that calculated by the original FS potential.

To correct these discrepancies, we adjust the FS potential at a fixed composition, using the following approach: DFT checks are performed after each GA search, and the DFT results are used to refine the FS potential by minimizing a weighted combination of the mismatches in energies, forces and stresses calculated by DFT and FS, using the POTFIT program $[43,44]$, Then a new GA search is performed with the refined FS 
potential. If structures with lower energies are found in the new GA search, these structures are added to the potential-refinement process. The above cycle is repeated until the GA search converges. About 30 structures for each motif (a total pool of over 300 structures) are used in the refinement process. This process is performed separately for a series of different $\mathrm{Cu}$ compositions to obtain accurate FS potentials over the whole stoichiometric range. The formation energies of the low-energy structures at $x_{\mathrm{Cu}}=0.65$ calculated by DFT and the refined FS potential are shown in Fig. 3 (b). Compared with Fig. 3 (a), the agreement between DFT and FS is greatly improved.

\section{Results and Discussions}

3.1 Energetic stability of medium-range motifs as a function of the composition

Besides $x_{\mathrm{Cu}}=0.65$, the iterative GA search is performed for several other $\mathrm{Cu}$ compositions $x_{\mathrm{Cu}}=0.33,0.5,0.69,0.73,0.78$, and 0.83 , where stable $\mathrm{Cu}-\mathrm{Zr}$ compounds exist. Low-energy structures as well as refined FS potentials are thus obtained at these compositions as well. We have provided the refined FS potentials in Supplementary Material. For each motif, we perform GA searches for $\mathrm{Cu}$ compositions ranging from 0.25 to 0.85 with interval of about 0.01 (the exact interval depends on the size of the supercell). At each composition, we use a FS potential interpolated from the refined potentials at nearby compositions. Figure 4 (a) shows the results of these extensive GA searches. DFT calculations are performed on the low-energy structures found in each GA search, and the formation energy is calculated based on the lowest DFT energy. Only the most stable crystalline-favoring motif at each $\mathrm{Cu}$ composition is shown in Fig. 4 (a) for easier viewing. At each $\mathrm{Cu}$ composition where there exists a line compound in the $\mathrm{Cu}-\mathrm{Zr}$ phase 
diagram in Fig. 4 (b), the crystallization-favoring motif characterized by the compound is found to be the most favorable structural order.

In the composition range $0.63<x_{C u}<0.68$, the Bergman-type icosahedral motif is the most stable medium-range order, and is responsible for glass formation due to its crystallization-resisting nature. This composition range compares favorably with one of the three optimal glass formation ranges observed in experiments [1]. On the other hand, no icosahedral motifs are stable outside of this composition range. Therefore, our results exclude icosahedral ordering as the fundamental reason for the glass formation at other composition ranges. Several other works that used the Voronoi tessellation technique to analyze the structures of $\mathrm{Cu}-\mathrm{Zr}$ glasses generated by molecular dynamics (MD) simulations also showed strong composition dependence of the icosahedral order [45-47]. The $\mathrm{Cu}$ composition for optimal icosahedral order established in such work varies from 0.65 to 0.75 , possibly due to the different interatomic potentials used in the MD simulations. The other icosahedral motifs are significantly less stable than the Bergman-type icosahedral motif, although they share similar short-range icosahedral clusters (except for the Tsai-type motif which has a tetrahedron core), indicating that medium-range order up to the second shell and beyond is important in the atomic arrangements of metallic glasses. At $0.63<x_{\mathrm{Cu}}<0.68$, the approximant structures used to model the Bergman-type icosahedral motif are unstable against phase separation into the equilibrium mixture of $\mathrm{Cu}_{10} \mathrm{Zr}_{7}$ and $\mathrm{Cu}_{8} \mathrm{Zr}_{3}$, since the formation energy of the Bergman approximant structures in this composition range [thick red line in Fig. 4 (a)] is above the tie line connecting the equilibrium $\mathrm{Cu}_{10} \mathrm{Zr}_{7}$ and $\mathrm{Cu}_{8} \mathrm{Zr}_{3}$ phases [dash-dotted line in Fig. 4 (a)]. This phase separation requires long-range transport of $\mathrm{Cu}$ and $\mathrm{Zr}$ atoms, 
which cannot be achieved under ultra-fast quenching conditions. Moreover, it also takes significant time for local Bergman clusters to arrange into a periodic network; thus, with fast quenching, even the approximant structures themselves can hardly be reached. However, since local atomic packing is a fast process, a significant fraction of local Bergman-type clusters can be formed given that they are energetically favorable.

\subsection{Effects of the configurational entropy}

The GA search that we have performed also allows us to estimate the configurational entropy originated from different decorations of the lattice structures. A large number of inequivalent decorations are generated during the course of a complete genetic-algorithm search. These structures form a reasonable pool for sampling the entire configurational space. Therefore, the free energy, including the contributions from the configurational entropy, can be estimated from the equation

$$
e^{-F / k_{B} T}=\sum_{i} e^{-E_{i} / k_{B} T}
$$

where $F$ is the free energy; $k_{B}$ is the Boltzmann constant; $T$ is the temperature; $E_{i}$ is the energy of each decoration; and $i$ sums over all inequivalent decorations visited during the GA search.

Taking the Bergman-type approximant structure as an example, over 10,000 structures with different atomic decorations are generated during the GA search for the ground state with the Bergman-type motif at $x_{\mathrm{Cu}} \sim 0.65$. Figure 5 (a) gives the density of states sampled from these structures, where it can be seen that the energies for these structures span multiple electronvolts per unit cell (containing 81 atoms). The large 
difference in energies for structures with different decorations is caused by the differential atomic size and chemical properties between the $\mathrm{Cu}$ and $\mathrm{Zr}$ elements. Most of these structures make negligible contributions to the free energy, since their energy separation from the ground state is much larger than $k_{B} T(\sim 0.03 \mathrm{eV}$ at room temperature). As a result, the total contribution from the configurational entropy, calculated by $F-E_{0} \quad\left(E_{0}\right.$ is the ground state energy), is only about $1 \mathrm{meV} /$ atom at room temperature, as shown in Fig. 5 (b). As shown in Fig. 4 (a), the structure representing the Bergman-type motif is at least $0.02 \mathrm{eV} /$ atom more stable than other structures at $x_{\mathrm{Cu}} \sim 0.65$. Therefore, the small contributions from the configurational entropy do not qualitatively affect the stability of the Bergman-type motif.

\subsection{Preference of Bergman MRO in rapidly cooled samples}

To verify that the Bergman-type order remains preferred under kinetic effects, we have performed Molecular Dynamics (MD) simulations on rapidly cooled $\mathrm{Cu}_{65} \mathrm{Zr}_{35}$ system. The unit cell contains 5,000 atoms with periodic boundary conditions. The refined FS potential at this composition is used for energy calculations. The samples are first melted and equilibrated at $T=1500 \mathrm{~K}$, and then cooled to $T=300 \mathrm{~K}$ at four different cooling rates: $5 \times 10^{12}, 5 \times 10^{11}, 5 \times 10^{10}$, and $5 \times 10^{9} \mathrm{~K} / \mathrm{s}$. To study the local order in a glassy sample, a cluster around each atom is extracted from the sample and aligned against an ideal template, using the method described in Ref. [13]. For the Bergman, Mackay and Tsai-type icosahedral motifs, the templates are given in the left panels of Fig. 1 (a)-(c), respectively, which all contain multiple atomic shells. For the MoAl-type, since the unit cell is made of a single icosahedron [see the left panel of Fig. 1 (d)], we choose two templates to represent two possible connections of neighboring icosahedra 
with a square or an octahedron, as shown in the right panel of Fig. 1 (d). In addition, the crystalline-favoring templates for the two crystalline phases with neighboring compositions $\mathrm{Cu}_{10} \mathrm{Zr}_{7}$ (Aba2) and $\mathrm{Cu}_{8} \mathrm{Zr}_{3}$ (Pnma) are also tested, in order to check whether the quenched sample contains nucleates of these two crystals. An alignment score, describing how an as-extracted cluster deviates from a perfect template, is defined as

$$
f=\left(\frac{\sum_{i=1}^{N}\left|r_{i c}-r_{i t}\right|^{2}}{N r_{0}^{2}}\right)^{1 / 2}
$$

where $N$ is the number of the atoms in the template, $r_{0}$ is the typical bond length of the template, $r_{i c}$ and $r_{i t}$ are the atom positions in the aligned cluster and template, respectively. The smaller the alignment score is, the less deviation the cluster has compared with the template. Figure 6 (a) shows the cumulative distributions of the alignment score obtained by aligning all the clusters in the sample prepared at the slowest cooling rate $5 \times 10^{9} \mathrm{~K} / \mathrm{s}$ against different templates. It is clear that the sample displays much better Bergman-type order than other types of icosahedral orders. The sample contains a very low population of $\mathrm{Aba} 2$ and Pnma type crystalline-favoring clusters, indicating that the glassy sample is free of nucleates of these crystalline phases. Figure 6 (b) plots the cumulative distribution of the alignment score against the Bergman template for samples cooled at different cooling rates. The fraction of Bergman-like clusters increases as the cooling rate decreases. Since our cooling rates are still much faster than those used in experiments, we expect actual fraction of Bergman clusters in real $\mathrm{Cu}-\mathrm{Zr}$ glasses to be even higher than our simulations. With the gradual enhancement of 
Bergman-type order as the cooling process is slowing down, the internal energy of the glass is also decreasing, as shown in the inset of Fig. 6 (b). This reconfirms that the Bergman-type motif is energetically favorable in the $\mathrm{Cu}-\mathrm{Zr}$ glass at $x_{\mathrm{Cu}} \sim 0.65$.

Several previous studies of CuZr-based glasses establish that the local icosahedral clusters up to the first shell are connected into an interpenetrating network [18, 48-50], in which two adjacent icosahedral clusters share a pentagonal bipyramid. The Bergman-type MRO also features similar interpenetrating connection. In an ideal Bergman motif [Fig. 1 (a)], a central icosahedral cluster is inter-connected to 12 outer icosahedral clusters, forming a "star-like" icosahedral suopercluster. Our molecular dynamics simulations show that a decreasing cooling rate promotes this connected Bergman order and enhances the icosahedral "star-like" network. Further clustering of Bergman motifs, which could provide useful information about the longer-range correlations of the system, can also be studied in MD simulations [14]. Unfortunately, to evaluate the energetic stablity of such clustering in the current GA-based method requires a unit cell containing multiple Bergman motifs, which exceeds the capability of global optimization-based structure prediction methods, even with the help of efficient interatomic potentials. (The difficulty of such problems grows exponentially with the system size).

\section{Conclusions}

In conclusion, we have developed a new method to characterize the stability of local medium-range order in metallic glasses with accurate first-principles calculations. This method enables us to clarify from a pure energetic point of view the origin for the selection of local structural order, which ultimately determines glass formation in deeply 
undercooled metallic liquids. The $\mathrm{Cu}-\mathrm{Zr}$ is an ideal model system to demonstrate the relative stability of various medium-range structural orders as a function of the composition. In the optimal glass-forming region with $x_{\mathrm{Cu}}$ around 0.65 , a crystallization-resisting Bergman-type motif is not only more stable than the motifs existing in stable $\mathrm{Cu}-\mathrm{Zr}$ compounds that are inclined to crystallization, but more stable than several other motifs with the same first-shell icosahedral order. Meanwhile, our results exclude icosahedral ordering as the fundamental reason for the glass formation in other composition ranges. A complete description of the $\mathrm{Cu}-\mathrm{Zr}$ glass is still pending on the identification of the non-icosahedral structural motifs for the other glass-forming composition ranges. However, the method outlined in the paper is complete and generally applicable to any system where appropriate candidate motifs are available.

\section{Acknowledgements}

Work at Ames Laboratory was supported by the US Department of Energy, Basic Energy Sciences, Division of Materials Science and Engineering, under Contract No. DE-AC02-07CH11358, including a grant of computer time at the National Energy Research Supercomputing Center (NERSC) in Berkeley, CA. F.Z. thank S.H. Zhou for preparing the $\mathrm{Cu}-\mathrm{Zr}$ phase diagram.

\section{References}

[1] Li Y, Guo Q, Kalb JA, Thompson CV. Science 2008;322:1816.

[2] Greer AL. Nature 1993;366:303.

[3] Turnbull D. Le Journal de Physique Colloques 1974;35:C4.

[4] Egami T, Waseda Y. J. Non·Cryst. Solids 1984;64:113. 
[5] Takeuchi A, Inoue A. JIM, Materials Transactions 2005;46:2817.

[6] Lu ZP, Liu CT, Wu Y, Tan H, Li Y, Chen GL. Intermetallics 2008;16:410.

[7] Weeber AW, Bakker H. Physica B: Condensed Matter 1988;153:93.

[8] Gallego LJ, Somoza JA, Alonso JA, Lopez JM. J. Phys. F: Met. Phys. 1988;18:2149.

[9] Xia L, Fang SS, Wang Q, Dong YD, Liu CT. Appl. Phys. Lett. 2006;88.

[10] Sheng H, Luo W, Alamgir F, Bai J, Ma E. Nature 2006;439:419.

[11] Luo W, Sheng H, Alamgir F, Bai J, He J, Ma E. Phys. Rev. Lett. 2004;92.

[12] Cheng YQ, Ma E. Appl. Phys. Lett. 2008;93:51910.

[13] Fang X, Wang C, Yao Y, Ding Z, Ho K. Phys. Rev. B 2010;82:184204.

[14] Fang XW, Wang CZ, Hao SG, Kramer MJ, Yao YX, Mendelev MI, Ding ZJ, Napolitano RE, Ho KM. Sci. Rep. 2011;1:194.

[15] Hwang J, Melgarejo ZH, Kalay YE, Kalay I, Kramer MJ, Stone DS, Voyles PM. Phys. Rev. Lett. 2012;108.

[16] Miracle DB. Nature Mater. 2004;3:697.

[17] Wang XD, Lou HB, Gong Y, Vainio U, Jiang JZ. J. Phys.: Condens. Matt. 2011;23:075402.

[18] Li M, Wang C, Hao S, Kramer M, Ho K. Phys. Rev. B 2009;80:184201.

[19] Hirata A, Guan P, Fujita T, Hirotsu Y, Inoue A, Yavari AR, Sakurai T, Chen M. Nat. Mater. 2011;10:28.

[20] Xu D, Lohwongwatana B, Duan G, Johnson WL, Garland C. Acta Mater. 2004;52:2621.

[21] Tang M-B, Zhao D-Q, Pan M-X, Wang W-H. Chin. Phys. Lett. 2004;21:901.

[22] Wang D, Li Y, Sun BB, Sui ML, Lu K, Ma E. Appl. Phys. Lett. 2004;84:4029. 
[23] Inoue A, Zhang W. Mech. Trans. 2004;45:584.

[24] Yu CY, Liu XJ, Lu J, Zheng GP, Liu CT. Sci. Rep. 2013;3:2124.

[25] Shintani H, Tanaka H. Nat. Phys. 2006;2:200.

[26] Goldman A, Kelton R. Rev. Mod. Phys. 1993;65:213.

[27] Tsai AP. Science and Technology of Advanced Materials 2008;9:013008.

[28] Okamoto H. Journal of phase equilibria and diffusion 2008;29:204.

[29] Bergman G, Waugh JLT, Pauling L. Acta Cryst. 1957;10:254.

[30] Cooper M, Robinson K. Acta Cryst. 1966;20:614.

[31] Palenzona A. Journal of the Less-Common metals 1971;25:367.

[32] Adam J, Rich JB. Acta Crystallogr. 1954;7:813.

[33] Mackay A. Acta Cryst. 1962;15:916.

[34] Tsai AP, Guo JQ, Abe E, Takakura H, Sato TJ. Nature 2000;408:537.

[35] Deaven D, Ho K. Phys. Rev. Lett. 1995;75:288.

[36] Ji M, Wang CZ, Ho KM. Physical Chemistry Chemical Physics 2010;12:11617.

[37] Finnis M, Sinclair J. Phil. Mag. A 1984;50:45.

[38] Mendelev MI, Kramer MJ, Ott RT, Sordelet DJ, Yagodin D, Popel P. Philos. Mag. 2009;89:967.

[39] Kresse G, Furthmüller J. Phys. Rev. B 1996;54:11169.

[40] Kresse G, Furthmüller J. Computational Materials Science 1996;6:15.

[41] Perdew JP, Burke K, Ernzerhof M. Phys. Rev. Lett. 1996;77:3865.

[42] Vanderbilt D. Phys. Rev. B 1990;41:7892.

[43] Peter B, Franz G. Modelling and Simulation in Materials Science and Engineering 2007;15:295.

[44] Brommer P, Gähler F. Philos. Mag. 2006;86:753. 
[45] Mendelev M, Kramer M, Ott R, Sordelet D, Besser M, Kreyssig A, Goldman A, Wessels V, Sahu K, Kelton K. Phil. Mag. 2010;90:3795.

[46] Ward L, Miracle D, Windl W, Senkov ON, Flores K. Phys. Rev. B 2013;88:134205.

[47] Wakeda M, Shibutani Y, Ogata S, Park J. Intermetallics 2007;15:139.

[48] Tomida T, Egami T. Phys. Rev. B 1995;52:3290.

[49] Hui X, Fang HZ, Chen GL, Shang SL, Wang Y, Liu ZK. Appl. Phys. Lett. 2008;92:201913.

[50] Wakeda M, Shibutani Y. Acta Materialia 2010;58:3963. 


\section{Figure Captions}

Fig. 1. Medium-range icosahedral order in typical quasicrystal approximants with bcc lattice. (a) $\operatorname{Mg}_{32}(\mathrm{Al}, \mathrm{Zn})_{49}$. Left: Bergman-type cluster inside the Wigner-Seitz unit cell, comprised of an icosahedron with the center occupied (red), a dodecahedron (blue), and a larger icosahedron (yellow). Right: Atoms on the surface of the unit cell including a 60-atom buckyball (green) and 12 additional atoms (pink) above 12 hexagons of the buckyball. (b) $\alpha(\mathrm{AlMnSi})$. Left: Mackay-type cluster inside the unit cell comprised of an icosahedron with the center vacant (red), a larger icosahedron twice the size of the first one (blue), and an icosidodecahedron (yellow). Right: atoms on the surface of the cell including 24 atoms on the edges of the truncated-octahedral unit cell (green), and 12 orange atoms close to the face diagonals of the squares. In $\alpha(\mathrm{AlMnSi})$, the orange atoms form a simple cubic lattice, that is, the body-centered counterparts are missing. However, when this structure is translated to the $\mathrm{Cu}-\mathrm{Zr}$ system, adding the missing atoms results in more stable structures. (c) $\mathrm{YbCd}_{6}$. Left: Tsai-type cluster inside the unit cell containing a central tetrahedron (red), a dodecahedron (blue), an icosahedron (yellow), and an icosidodecahedron (cyan). Right: atoms on the surface of the unit cell, similar to that in (b). (d) $\mathrm{MoAl}_{12}$. Left: MoAl-type cluster which is an icosahedron with the center occupied. Right: the connection of two neighboring icosahedra through a square (MoAl-Sq.) and an octahedron (MoAl-Octa.) along the (001) and (111) directions, respectively.

Fig. 2. Schematic illustration of the "cut-and-paste" mating operation in the GA search. In the current study, both parent structures have the same Bravais lattice and atomic sites, but have different atomic decorations. To perform the mating operation, we first create two bounding planes (dashed lines) to cut the parent structures into separate regions 
denoted by different colors. Then, a child structure is assembled by combining the atoms in the regions of the two parent structures that have the same color. Since this "cut-and-paste" operation can result in a different atomic composition from the desired value, if necessary, we finally apply a series of flipping operations (i.e., randomly changing a $\mathrm{Cu}$ atom to $\mathrm{Zr}$ and vice versa) to correct the composition.

Fig. 3. Comparison of the formation energies calculated by (a) the original FS potential and (b) the refined FS potential with those calculated by DFT for the low-energy structures found in GA searches at $x_{\mathrm{Cu}} \sim 0.65$.

Fig. 4. (a) Formation energy of the lowest energy structure for each motif found in GA search, as a function of $x_{\mathrm{Cu}}$. At each composition, only the most stable crystallization-favoring motif is shown for easier viewing. The bold red line highlights the composition range where the crystallization-resisting Bergman-type icosahedral motif is more energetically favorable than all crystallization-favoring motifs. (b) The phase diagram of the $\mathrm{Cu}-\mathrm{Zr}$ system.

Fig. 5. (a) Density of states for all the structure visited during the genetic-algorithm search for the optimal Bergman-type approximant structure at $x_{\mathrm{Cu}} \sim 0.65$. The unit cell contains 81 atoms. The energy of the ground state is set to be zero. (b) $F-E_{0}$ as a function of the temperature, where $F$ and $E_{0}$ refer to the free energy and the ground-state energy, respectively. 
Fig. 6. (a) The cumulative distribution of the alignment score against different types of icosahedral templates for the sample prepared at a cooling rate of $5 \times 10^{9} \mathrm{~K} / \mathrm{s}$. (b) The cumulative distribution of the alignment score against the Bergman template for samples prepared at different cooling rates. Inset: the internal energy of the sample at $300 \mathrm{~K}$ as a function of the cooling rate. 

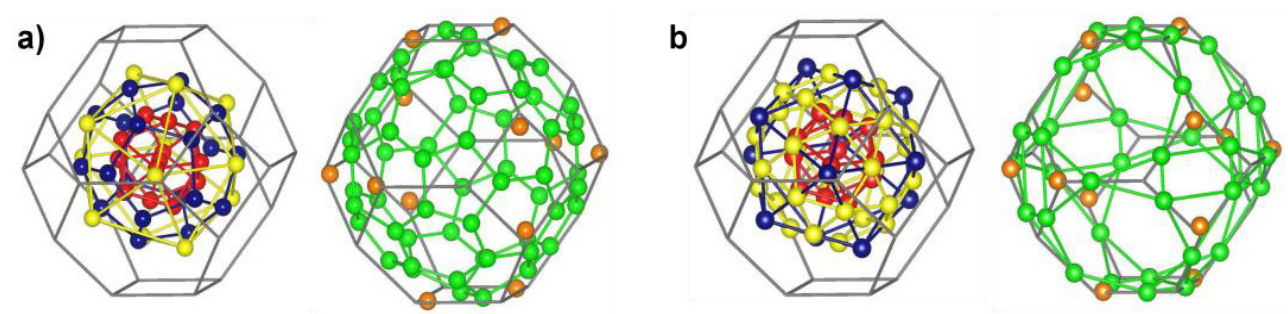

c)
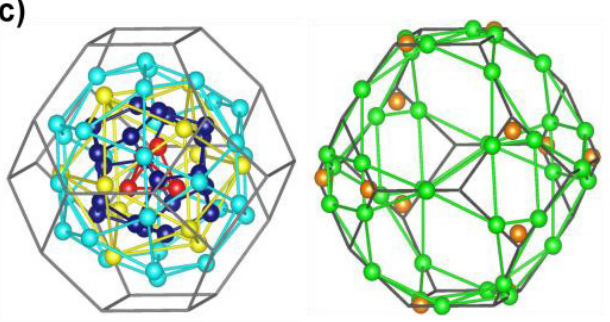

d)

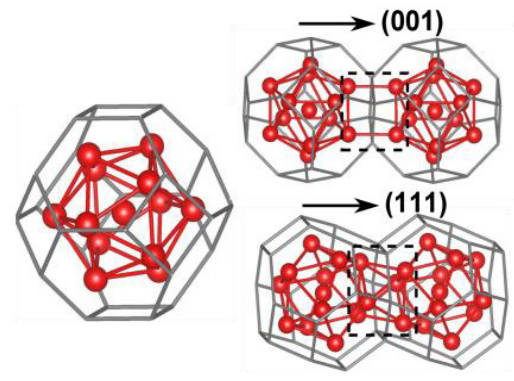

Fig. 1. Medium-range icosahedral order in typical quasicrystal approximants with bcc lattice. (a) $\operatorname{Mg}_{32}(\mathrm{Al}, \mathrm{Zn})_{49}$. Left: Bergman-type cluster inside the Wigner-Seitz unit cell, comprised of an icosahedron with the center occupied (red), a dodecahedron (blue), and a larger icosahedron (yellow). Right: Atoms on the surface of the unit cell including a 60atom buckyball (green) and 12 additional atoms (pink) above 12 hexagons of the buckyball. (b) $\alpha(\mathrm{AlMnSi})$. Left: Mackay-type cluster inside the unit cell comprised of an icosahedron with the center vacant (red), a larger icosahedron twice the size of the first one (blue), and an icosidodecahedron (yellow). Right: atoms on the surface of the cell including 24 atoms on the edges of the truncated-octahedral unit cell (green), and 12 orange atoms close to the face diagonals of the squares. In $\alpha(\mathrm{AlMnSi})$, the orange atoms form a simple cubic lattice, that is, the body-centered counterparts are missing. However, when this structure is translated to the $\mathrm{Cu}-\mathrm{Zr}$ system, adding the missing atoms results in more stable structures. (c) $\mathrm{YbCd}_{6}$. Left: Tsai-type cluster inside the unit cell containing a central tetrahedron (red), a dodecahedron (blue), an icosahedron (yellow), and an icosidodecahedron (cyan). Right: atoms on the surface of the unit cell, similar to that in 
(b). (d) MoAl $1_{12}$. Left: MoAl-type cluster which is an icosahedron with the center occupied.

Right: the connection of two neighboring icosahedra through a square (MoAl-Sq.) and an octahedron (MoAl-Octa.) along the (001) and (111) directions, respectively. 

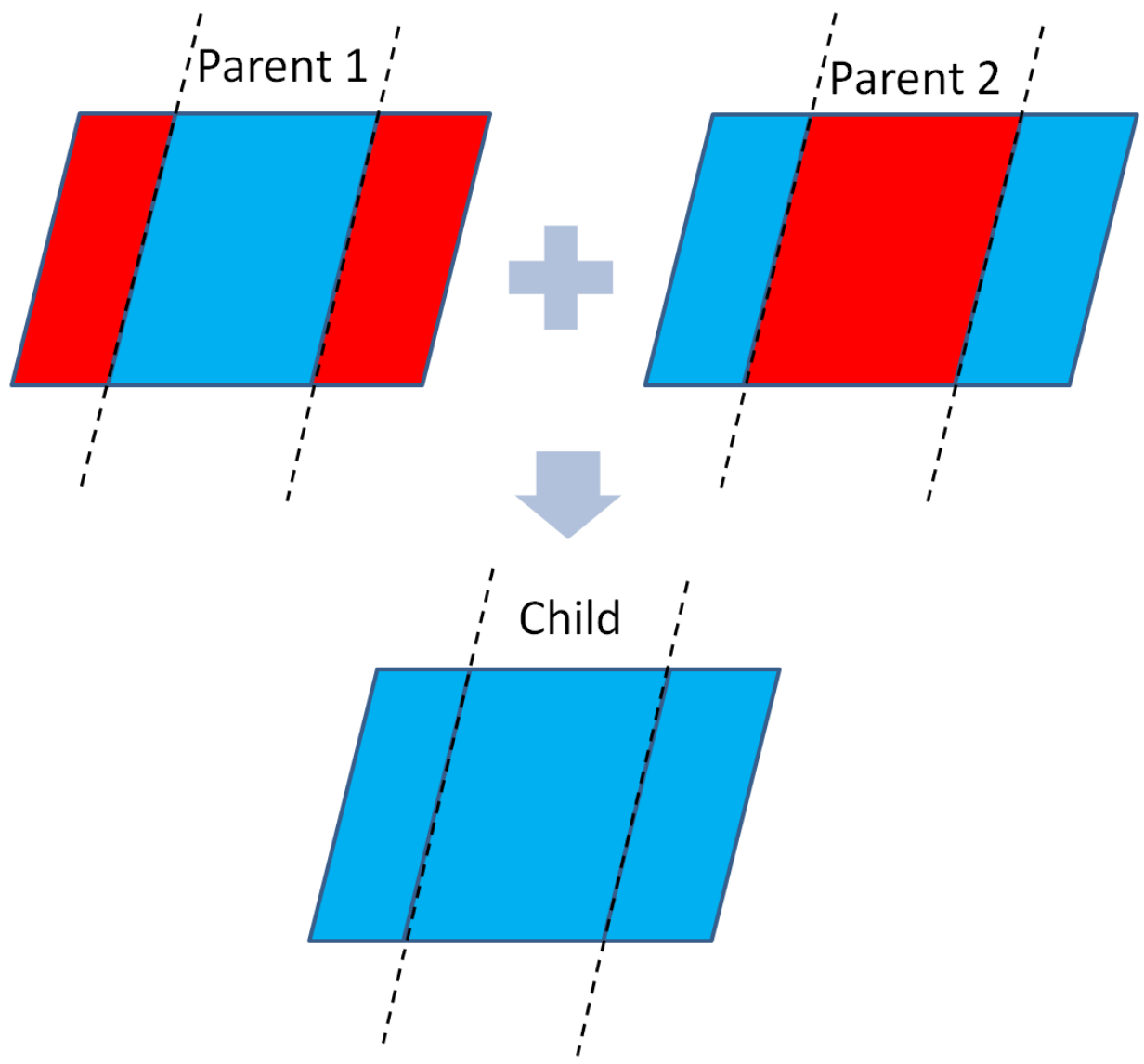

Fig. 2. Schematic illustration of the "cut-and-paste" mating operation in the GA search. In the current study, both parent structures have the same Bravais lattice and atomic sites, but have different atomic decorations. To perform the mating operation, we first create two bounding planes (dashed lines) to cut the parent structures into separate regions denoted by different colors. Then, a child structure is assembled by combining the atoms in the regions of the two parent structures that have the same color. Since this "cut-andpaste" operation can result in a different atomic composition from the desired value, if necessary, we finally apply a series of flipping operations (i.e., randomly changing a $\mathrm{Cu}$ atom to $\mathrm{Zr}$ and vice versa) to correct the composition. 

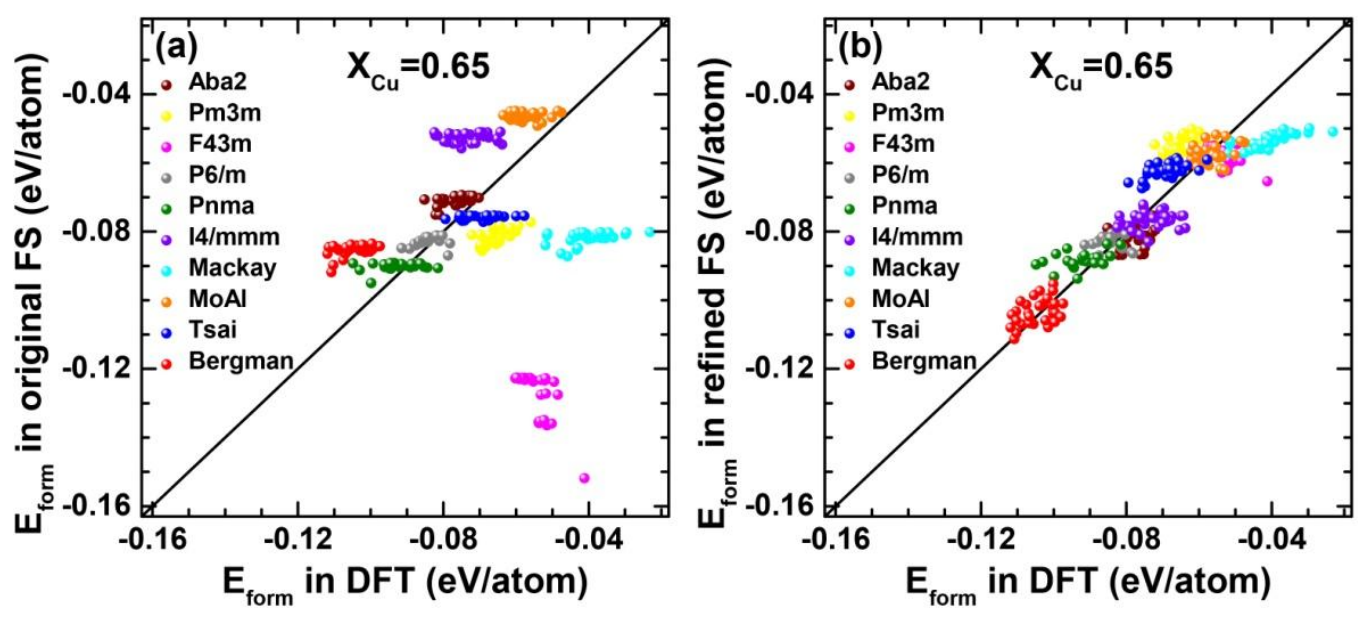

Fig. 3. Comparison of the formation energies calculated by (a) the original FS potential and (b) the refined FS potential with those calculated by DFT for the low-energy structures found in GA searches at $x_{\mathrm{Cu}} \sim 0.65$. 


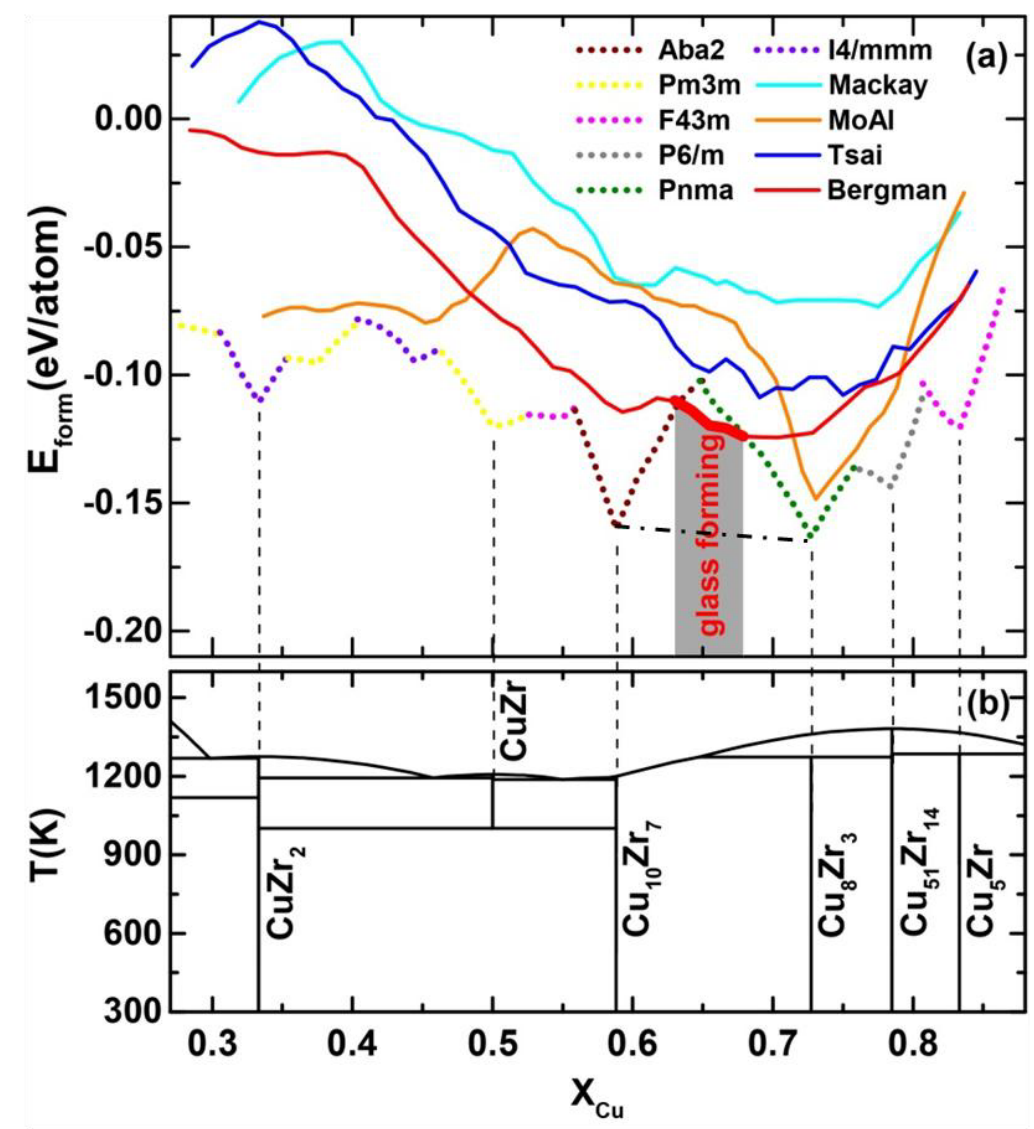

Fig. 4. (a) Formation energy of the lowest energy structure for each motif found in GA search, as a function of $x_{\mathrm{Cu}}$. At each composition, only the most stable crystallizationfavoring motif is shown for easier viewing. The bold red line highlights the composition range where the crystallization-resisting Bergman-type icosahedral motif is more energetically favorable than all crystallization-favoring motifs. The dash-dotted line is the tie line connecting the thermodynamically equilibrium $\mathrm{Cu}_{10} \mathrm{Zr}_{7}$ and $\mathrm{Cu}_{8} \mathrm{Zr}_{3}$ phases. (b) The phase diagram of the $\mathrm{Cu}-\mathrm{Zr}$ system. 

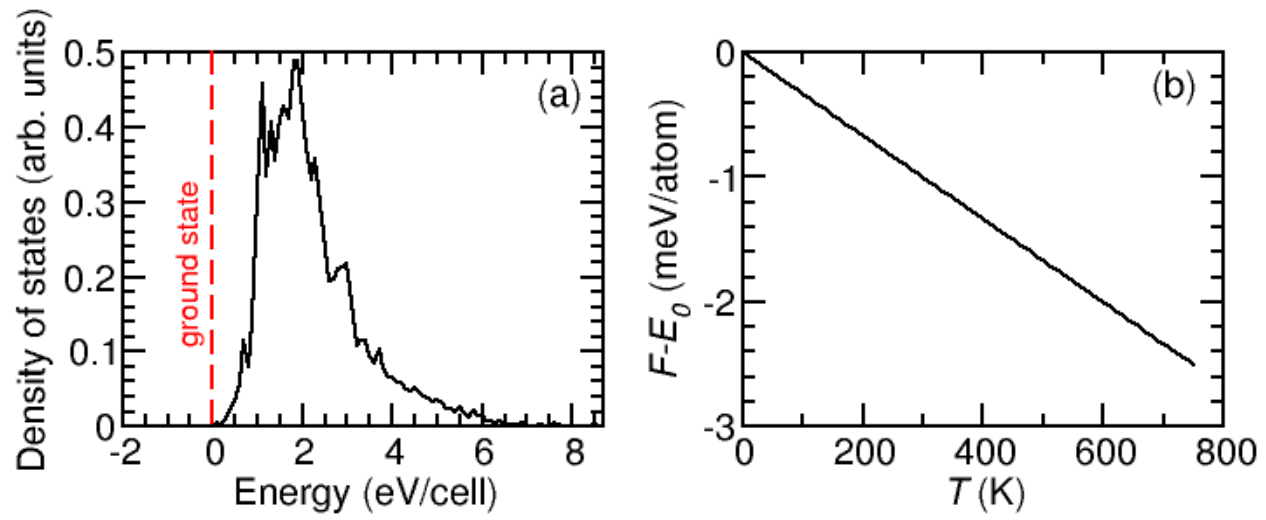

Fig. 5. (a) Density of states for all the structure visited during the genetic-algorithm search for the optimal Bergman-type approximant structure at $x_{\mathrm{Cu}} \sim 0.65$. The unit cell contains 81 atoms. The energy of the ground state is set to be zero. (b) $F-E_{0}$ as a function of the temperature, where $F$ and $E_{0}$ refer to the free energy and the ground-state energy, respectively. 


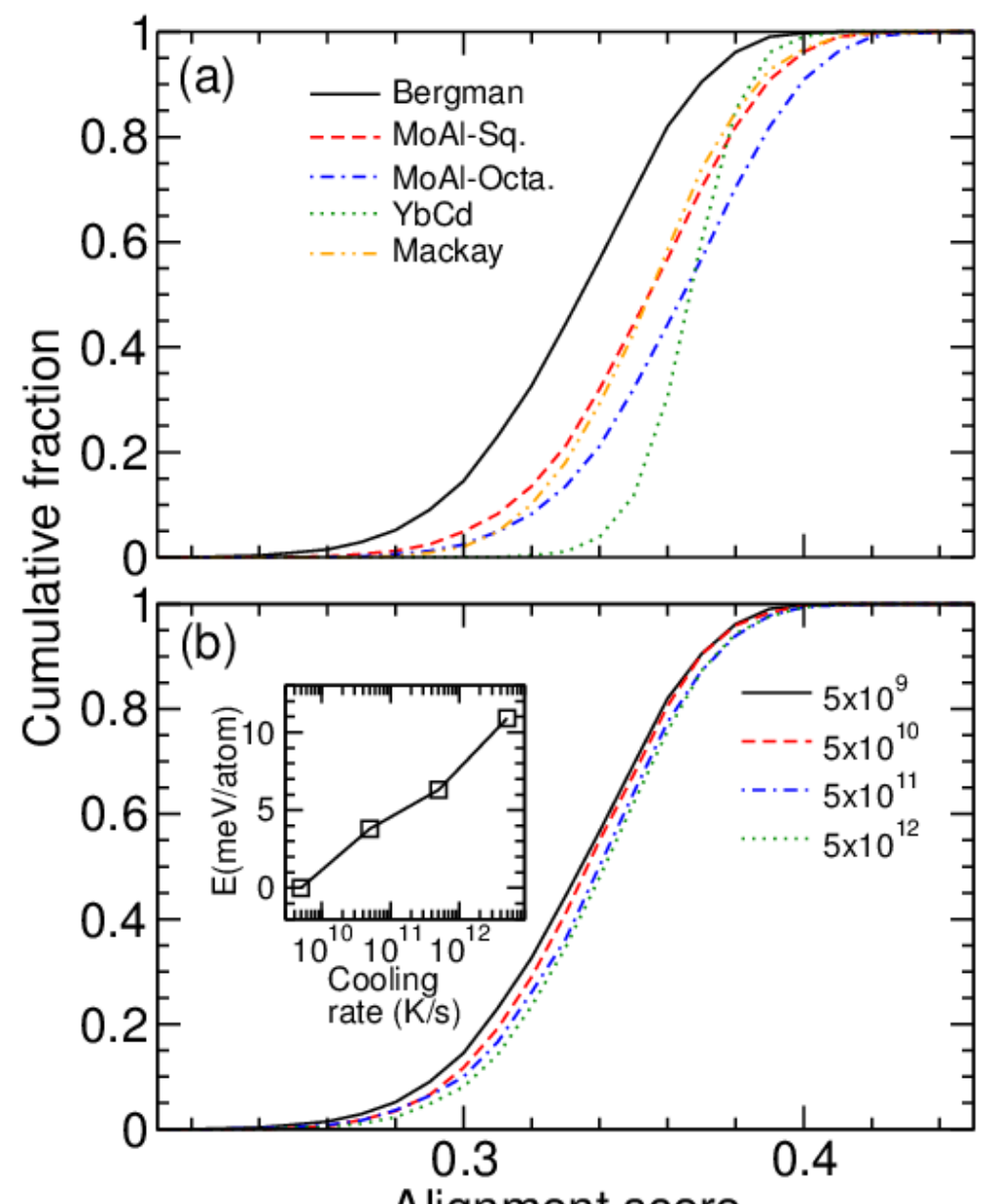

Alignment score

Fig. 6. (a) The cumulative distribution of the alignment score against different types of icosahedral templates for the sample prepared at a cooling rate of $5 \times 10^{9} \mathrm{~K} / \mathrm{s}$. (b) The cumulative distribution of the alignment score against the Bergman template for samples prepared at different cooling rates. Inset: the internal energy of the sample at $300 \mathrm{~K}$ as a function of the cooling rate. 